\title{
Gamma-ray attenuation technique for measuring void fraction in horizontal gas-liquid two-phase flow
}

\author{
LI Zhibiao* WU Yingxiang LI Donghui \\ (Institute of Mechanics, the Chinese Academy of Sciences, Beijing100080, China)
}

\begin{abstract}
The measurement of void fraction is of importance to the oil industry and chemical industry. In this article, the principle and mathematical method of determining the void fraction of horizontal gas-liquid flow by using a single-energy $\gamma$-ray system is described. The $\gamma$-ray source is the radioactive isotope of ${ }^{241} \mathrm{Am}$ with $\gamma$-ray energy of 59.5 keV. The time-averaged value of the void fraction in a $50.0-\mathrm{mm}$ i.d. transparent horizontal pipeline is measured under various combinations of the liquid flow and gas flow. It is found that increasing the gas flow rate at a fixed liquid flow rate would increase the void fraction. Test data are compared with the predictions of the correlations and a good agreement is found. The result shows that the designed $\gamma$-ray system can be used for measuring the void fraction in a horizontal gas-liquid two-phase flow with high accuracy.
\end{abstract}

Keywords $\gamma$-rays, Single-energy, Void fraction, Two-phase flow

CLC number TP391

\section{Introduction}

The imaging and measurement of multiphase flows have received much attention in recent years, largely driven by the need in the oil industry to measure the mass flow rates of oil, water, and gas in the production pipelines. The ability to see the inside of an object and make quantitative measurements of the enclosed materials and structures has a wide range of applications. However, the measurement over a wide range of flow regimes and the ability to measure mass flow rates of each component with high accuracy require a detailed knowledge of the hydrodynamics of multiphase flows, especially the phase fraction on the cross-section of a pipeline for each component, as the fast changing phase fractions directly control the multiphase flow behavior and flow rates, and also the basic information to reconstruct the flow pattern images. Therefore, in recent years many researchers have devoted their attention to determining phase fractions and to improving phase fraction measurement accu- racy. Daidzic et al. ${ }^{[1]}$ have measured void fraction using the magnetic resonance imaging (MRI) system. The shortcoming of this approach is that the liquid should not be conductible. Kendoush and Sarkis ${ }^{[2]}$ have measured the void fraction using X-ray absorption. Yang et al. ${ }^{[3]}$ have measured the void fraction using the impedance method. Wojtan et al. ${ }^{[4]}$ have measured the dynamic void fraction in stratified types of flow. Harms et al. ${ }^{[5]}$ have proposed a void fraction model for annular flow in a horizontal pipe.

Radiation technique is being considered as a better option to get the details of a multiphase flow structure, as information about phase distribution can be obtained destructively. Stahl et al. ${ }^{[6]}$ have discussed the accuracy of void fraction measurements by single-beam gamma-densitometry. Takenaka et al. ${ }^{[7]}$ have measured the void fraction by neutron radiography. The motivation for the study presented in this article was to examine how $\gamma$-rays could be used in a particular field of industrial imaging, that is, the imaging and measurement of gas-liquid two-phase flows in pipe-

Supported by National Natural Science Foundation of China (No. 10572143)

*E-mail: zhibiaoli@163.com

Received date: $2007-01-04$ 
lines. Specifically, the possible role of $\gamma$-ray techniques in an application of oil industry that requires figuring out the phase fraction, by accurately measuring flow rates of liquid and gas in the oil production pipes should be examined.

The radiation technique considered in this study is associated with a single-energy $\gamma$-ray system. Liquid in the test section attenuate $\gamma$-radiation without depositing significant amounts of energy. As the gas phase has little attenuating power for $\gamma$-radiation, the attenuation of gas can be neglected. Therefore, by detecting the attenuation of the $\gamma$-radiation beam, the phase fraction in the flow channel can be measured. In this article, the aim is to apply the single-energy $\gamma$-ray radiation technique to determine the cross-section void fraction in a horizontal gas-liquid two-phase flow.

\section{Measurement principle}

Attenuation of $\gamma$-rays through a sample of thickness $L$ is given by

$$
\ln \left(\frac{I}{I_{0}}\right)=-\mu L
$$

where $\mu$ is the mean linear attenuation coefficient of the sample, $I_{0}$ represents the incident (upon the sample) intensity of the $\gamma$-ray flux, $I$ is the outgoing intensity from the sample and $L$ is the sample thickness. When the $\gamma$-ray passes through different materials, the relevant relations become

$$
\ln \left(\frac{I}{I_{0}}\right)=-\mu_{1} L_{1}-\mu_{2} L_{2}-L
$$

where subscripts $1,2, \ldots$ denote the materials with different linear attenuation coefficients.

According to Eq.(2), when the $\gamma$-ray passes through the object composed of water and air, the intensity attenuation yields:

$$
\left\{\begin{array}{l}
\mu_{\mathrm{w}} L_{\mathrm{w}}+\mu_{\mathrm{a}} L_{\mathrm{a}}=-\ln \left(\frac{I}{I_{0}}\right) \\
L_{\mathrm{w}}+L_{\mathrm{a}}=d
\end{array}\right.
$$

where subscripts $w$ and a denote the material of water and air, respectively, $d$ is the pipe diameter.

In this study, $I_{0}$ represents the intensity of the $\gamma$-ray beam after it passes through the upper wall, and $I$. represents the intensity of the $\gamma$-ray beam before it passes through the bottom wall. The attenuation of air is so small that it can be neglected. Hence, Eq.(3) can be written as

$$
\left\{\begin{array}{l}
\mu_{w} L_{w}=-\ln \left(\frac{I}{I_{0}}\right) \\
L_{a}=d-L_{w}
\end{array}\right.
$$

By solving Eq.(4), the thickness of air in the test section can be obtained. So, the void fraction can be written as

$$
\alpha=\left\{\begin{array}{l}
\frac{1}{\pi} \arccos \left(1-\frac{2 L_{\mathrm{a}}}{d}\right)-\frac{2\left(d-L_{\mathrm{a}}\right) \sqrt{L_{\mathrm{a}}\left(d-L_{\mathrm{a}}\right)}}{\pi d^{2}},\left(L_{\mathrm{a}}<\frac{d}{2}\right) \\
1-\frac{1}{\pi} \arccos \left(-1+\frac{2 L_{\mathrm{a}}}{d}\right)+\frac{2 L_{\mathrm{a}} \sqrt{L_{\mathrm{a}}\left(d-L_{\mathrm{a}}\right)}}{\pi d^{2}},\left(L_{\mathrm{a}}>\frac{d}{2}\right)
\end{array}\right.
$$

\section{Experimental system}

\subsection{Experimental setup}

In this study, the $\gamma$-ray source is the radioactive isotope ${ }^{241} \mathrm{Am}$, which emits $\gamma$-ray with energies of 59.5 $\mathrm{keV}$. The radioactive isotope is assembled and shielded in a thick lead pot to prevent the harmful emission of ${ }^{241} \mathrm{Am} \gamma$-rays. The radiation activity of the isotope is $3.7 \mathrm{GBq}$. A collimated single $\gamma$-ray beam (in $20 \mathrm{~mm}$ diameter) radiates from the bottom of the source pot and can be turned on/off by a mechanical switch, to ensure operation safety (see Fig.1).



Fig.1 Experimental setup.

The scintillation detector is made of NaI crystal. A column crystal of scintillator with a size of $40 \mathrm{~mm}$ (height) $\times 40 \mathrm{~mm}$ (diameter) is connected with a photomultiplier tube. The total diameter of the detector is $55 \mathrm{~mm}$ and the length is $220 \mathrm{~mm}$. In addition, a collimation hole with a size of $50 \mathrm{~mm} \times 30 \mathrm{~mm} \times 150 \mathrm{~mm}$ 
(length $x$ width $\times$ height) is mounted on top of the detector (see Fig.1).

The nuclear instrument presented in this study is designed as a multi-channel type. The system is operated in count mode. It is made up of high voltage power supply, amplifier, shaping amplifier, and programmable data acquisition system.

The experiment was carried out in the Multiphase Flow Laboratory of the Institute of Mechanics, Chinese Academy of Sciences (see Fig.1). The length of the test section is $10 \mathrm{~m}$ and the plexiglas pipe inner diameter is $50 \mathrm{~mm}$. The superficial velocities of air and water are $0.075 \sim 1.248 \mathrm{~m} / \mathrm{s}$ and $0.539 \sim 1.617 \mathrm{~m} / \mathrm{s}$, respectively.

When the pipe is empty, from Eq.(1),

$$
\ln \left(\frac{I_{2}}{I_{1}}\right)=-2 \mu_{\mathrm{p}} L_{\mathrm{p}}
$$

where $I_{2}$ and $I_{1}$ represent the intensity of the $\gamma$-ray beam after and before it passes through the test section, respectively, p denotes the pipe. So, the value of $I$ and $I_{0}$ can be obtained:

$$
\begin{aligned}
& I_{0}=I_{1} / \exp \left(\mu_{\mathrm{p}} L_{\mathrm{p}}\right) \\
& I=I_{2} \exp \left(\mu_{\mathrm{p}} L_{\mathrm{p}}\right)
\end{aligned}
$$

From Eqs. (7), (4), and (5), the void fraction can be calculated.

\subsection{Static calibration}

Table 1 is the static calibration data, which shows that the system can be used to measure the void fraction accurately in static conditions.

Table 1 Static calibration

\begin{tabular}{lll}
\hline $\begin{array}{l}\text { Actual void } \\
\text { fraction }\end{array}$ & $\begin{array}{l}\text { Measured void } \\
\text { fraction }\end{array}$ & Relative error/\% \\
\hline 0.10408 & 0.10463 & 0.53324 \\
0.26621 & 0.26308 & -1.17689 \\
0.52772 & 0.52708 & -0.12166 \\
0.7343 & 0.72922 & -0.69222 \\
0.9099 & 0.9075 & -0.26409 \\
\hline
\end{tabular}

\section{Results and discussion}

\subsection{Experimental data}

The relation between void fraction and mixture velocity are shown in Fig.2. $X$-axis denotes mixture velocity and $Y$-axis denotes void fraction. HVF stands for the prediction of the homogeneous model, and MVF is the measured void fraction. From Fig.2, it can be seen that void fraction increases with the increasing of mixture velocity. MVF and HVF have the same tendency, but at high mixture velocity MVF is less than HVF. The reason for this fact can be explained as follows. According to the assumption of the homogeneous model, gas and liquid have the same velocity in the pipe flow. But the real situation is, when the superficial velocity of gas is increased, actual velocity of gas is larger than actual velocity of liquid, so the slip velocity between gas and liquid causes MVF to be less than HVF at high mixture velocity. The superficial velocities of the liquid in Fig.2 (a) and (b) are 0.8985 $\mathrm{m}^{-1} \mathrm{~s}^{-1}$ and $1.6173 \mathrm{~m} / \mathrm{s}$, respectively. The maximum relative error between MVF and HVF is less than $10 \%$.
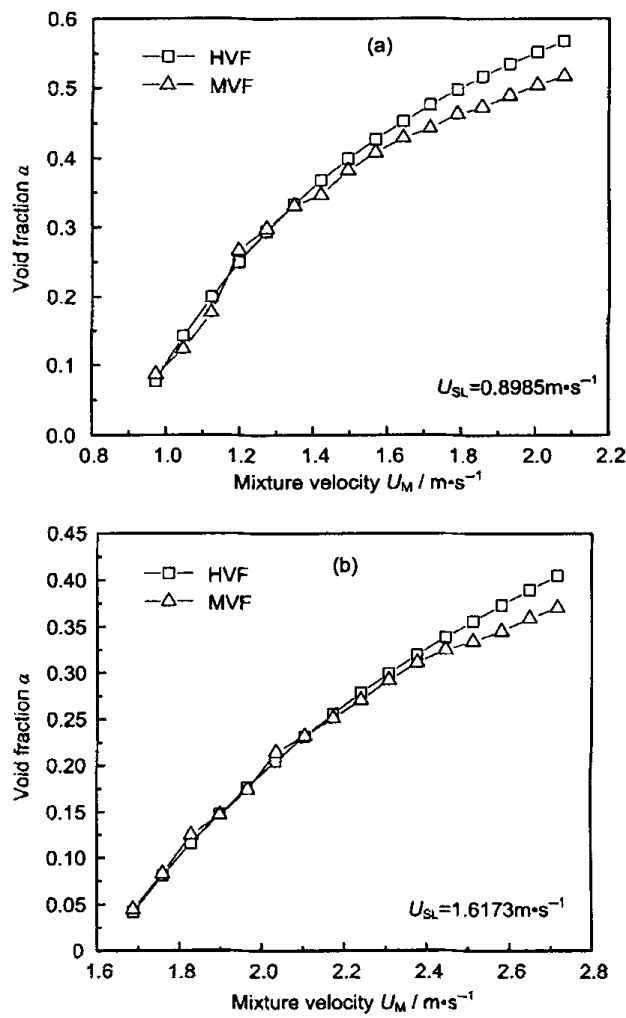

Fig.2 Comparison of the test data with the homogeneous model.

\subsection{Comparison with other correlations}

Armand ${ }^{[8]}$ proposed an empirical correlation:

$$
\alpha=\frac{1}{1+\left(0.2+1.2 /\left(U_{\mathrm{sG}} / U_{\mathrm{SL}}\right)\right)}
$$


This correlation has been widely used for the calculation of void fraction in horizontal gas-liquid two-phase flows. Modified Armand equation was proposed based on the test data:

$$
\alpha=\frac{1}{1+\left(0.2+0.98574 /\left(U_{S G} / U_{S L}\right)\right)}
$$

The result is shown in Fig.3 (a). The $X$-axis denotes the ratio of the superficial velocity of gas to the superficial velocity of liquid, and the $Y$-axis denotes the void fraction. The relative error is less than $8 \%$.
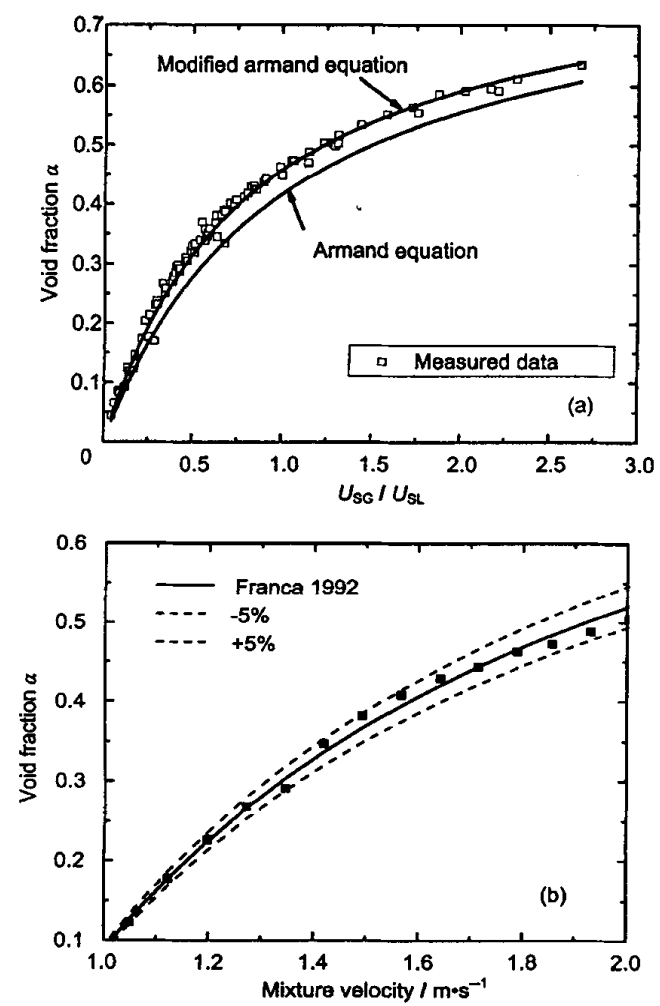

Fig.3 Comparison of the test data with correlation of Armand and Franca.

Franca et al. ${ }^{[9]}$ measured the void fraction using a quick valve in the horizontal pipe, and proposed an empirical correlation of the void fraction:

$$
\alpha=\frac{U_{\mathrm{SG}}}{0.98 U_{\mathrm{M}}+0.16}
$$

The comparison between the test data and the predicted value is shown in Fig.3 (b). The maximum relative error is $5 \%$. The comparison of the test data with the correlations of Armand and Franca shows that the $\gamma$-ray system designed here can be used for measuring the void fraction in horizontal gas-liquid two-phase flow with high accuracy.

\section{Conclusions}

The measurement of the void fraction is of considerable importance to the oil and chemical industries. The principle and mathematical method of determining the void fraction of a horizontal gas-liquid flow, by using a single-energy $\gamma$-ray system is described.

The time-averaged value of a void fraction in a 50.0-mm i.d. transparent horizontal pipeline was measured. The measurements were made at various combinations of the liquid flow and gas flow. It was found that increasing the gas flow rate at a fixed liquid flow rate would increase the void fraction. Test data were compared with the predictions of the following correlations:

(1) The maximum relative error between test data and homogeneous model prediction was less than $10 \%$.

(2) Test data had good agreement with the Armand equation. ${ }^{[8]}$

(3) The maximum relative error between the test data and Franca correlation prediction ${ }^{[9]}$ was $5 \%$.

\section{Acknowledgments}

The authors acknowledge Wang Keren, $\mathrm{Ma}$ Naiqing, Yuan Maozhu and Ma Runhai for their collaboration and suggestions in the phase fraction determination.

\section{References}

1 Daidzic N E, Schmidt E, Hasan M M et al. Nuclear Engineering and Design, 2005, 10: 1163-1178.

2 Kendoush A A, Sarkis Z A. Experimental Thermal and Fluid Science, 2002, 25: 615-621.

3 Yang H C, Kim D K, Kim MH. Flow Measurement and Instrumentation, 2003, 14: 151-160.

4 Wojtan L, Ursenbacher T, Thome J R. Experimental Thermal and Fluid Science, 2005, 29: 383-392.

5 Harms T M, Li D Q, Grol E A, et al. Int J Heat and Mass Transfer, 2003, 46: 4051-4057.

6 Stahl P, von Rohr P R. Experimental Thermal and Fluid Science, 2004, 28: 533-544

7 Takenaka N. Asano H. Experimental Thermal and Fluid Science, 2005, 29: 393-402.

8 Armand A A. Izv Vses Teplotekh Inst, 1946, 1: 16-23. AERE Trans. No. 828.

9 Franca F, Lahey R T Jr. Int J Multiphase Flow, 1992, 6: 787-801. 